\title{
Minimally Invasive Image Guided Interventions in Gynecology and Women's Health
}

\author{
Arindam Bharadwaz* \\ Senior Consultant, Aarhus University Hospital, Denmark
}

*Corresponding author: Arindam Bharadwaz, Senior Consultant, President, Danish Society of Interventional Radiology, Aarhus University Hospital, Denmark

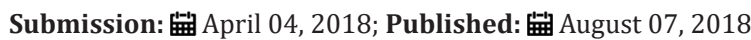

\section{Introduction}

Image guided interventions are increasingly being used in different fields of medicine. A large number of such minimally invasive interventions are routinely done for surgical, medical and oncological patients, besides the endovascular interventions performed for a variety of vascular conditions and diseases. Minimally invasive image guided interventions in gynaecology are primarily embolization procedures where supplying arteries or draining veins are occluded using different embolization materials. The major image guided interventions are described below (Figure 1).

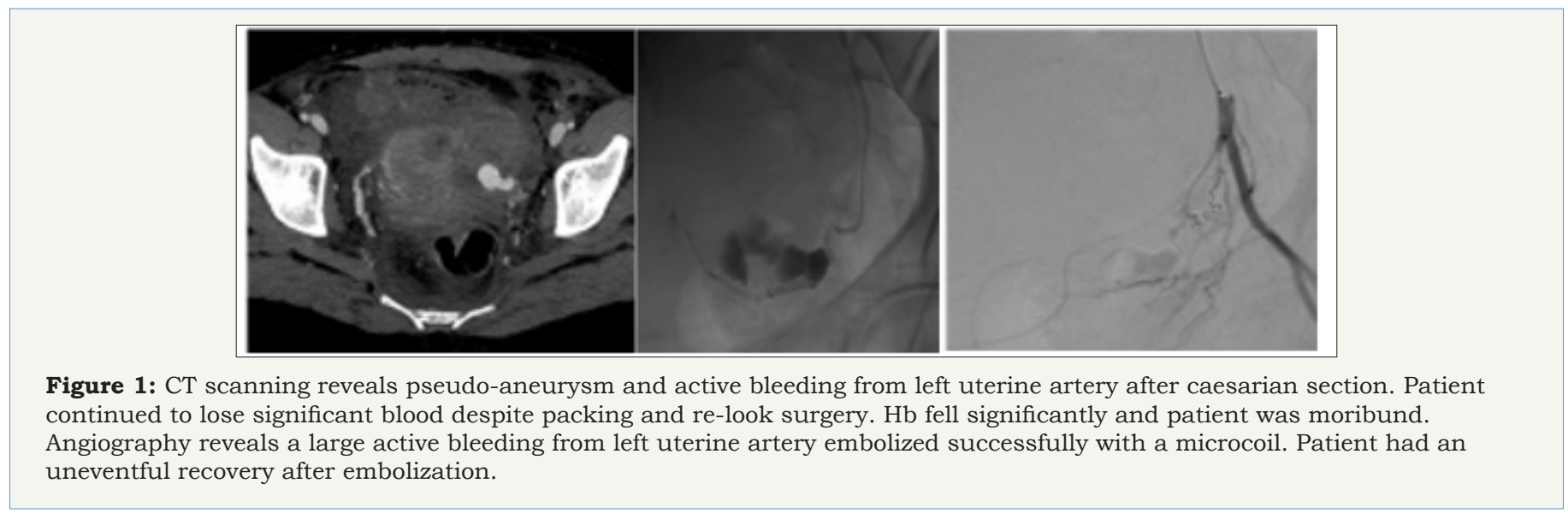

\section{Hemorrhage}

Uterine and pelvic arterial embolization is a life saving tool for post partum hemorrhage (PPH) [1,2], post-operative bleeding including after caesarian section and hysterectomy, and for bleeding (or risk of bleeding) in abnormal placentation, post-abortion/ ectopic pregnancy, trophoblastic disorders and gynecological malignancies [3]. The embolization techniques are minimally invasive, quick and easy to perform saves a lot of blood transfusion and patients recover early with shorter hospital stay. Most embolizations are performed through a puncture in the right and/or left common femoral artery and use 4F-5F selective catheters, co-axial micro-catheters $(2.4 \mathrm{~F}-3 \mathrm{~F})$ and corresponding guide-wires. Typical embolization materials used to occlude the bleeding vessels are coils (steel, platinum, fibred or hydro gel coils), particles (uncalibrated Gelfoam, calibrated particles from 500-900 micron) and liquid embolic materials (Glue \& Onyx). Coils and Gelfoam are the most frequently used embolic agents.

\section{Uterine fibroids}

Leiomyomas occur in $50-60 \%$ of women, rising to $70 \%$ by the age of 50 [4]. Theycause pressure symptoms such urinary symptoms, heaviness, pelvic pain, infertility and obstetric complications, and in $30 \%$ of cases, cause heavy menstrual bleeding leading to anemia [5]. Effectiveness of uterine artery embolization (UAE) in treating selected patients with uterine fibroids has been established by multiple studies. Royal College of Obstetrics \& Gynecologists (RCOG) recommends that UAE should always be considered along with surgical options [6]. UAE has similar outcome as surgery in up to a five-year follow up with no greater major complication rate [7]. On the other hand UAE is minimally invasive, can be performed on a day-care basis and thus has very short hospital stay compared to surgery, though with a higher re-intervention rate in the long term. Pre-treatment and follow up MR scanning is mandatory to diagnose, plan intervention and evaluate treatment success and rule out complications. UAE is always performed bilaterally as 
leiomyomas tend to have bilateral uterine arterial supply. 500-900 micron particles are used for embolization until complete stasis of the uterine arteries. Smaller particles are usually not recommend- ed to avoid complications such as necrosis of fibroid/ uterus. Cervical branches should also be avoided during UAE (Figure 2 \& 3).
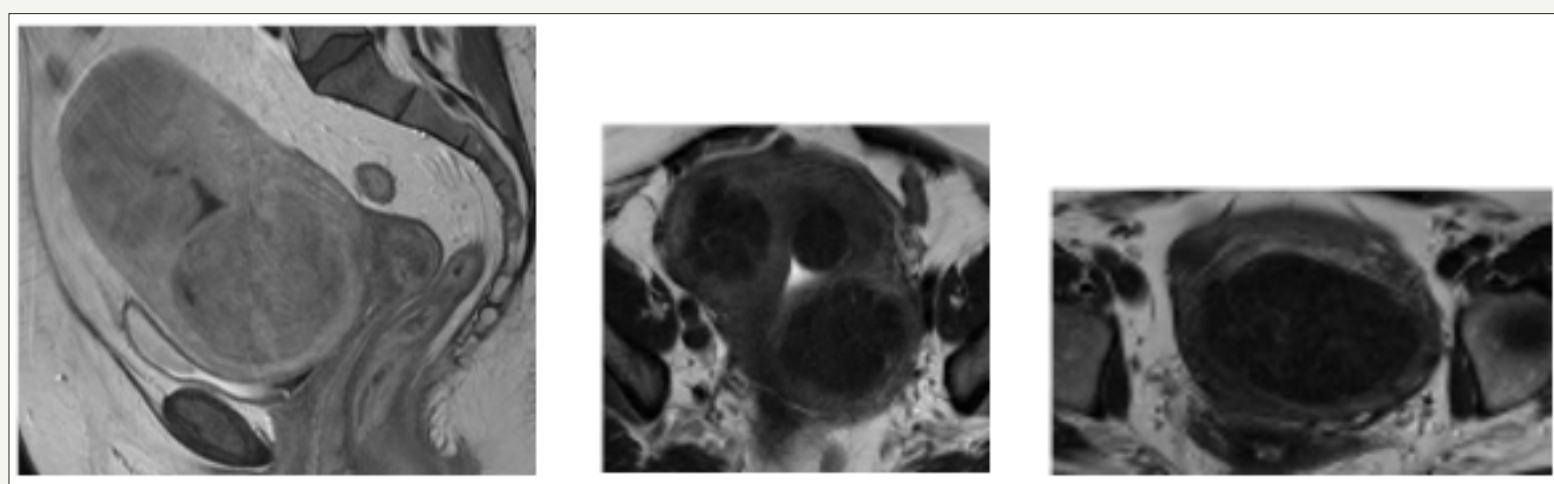

Figure 2: MR images show multiple large fibroids in uterus.

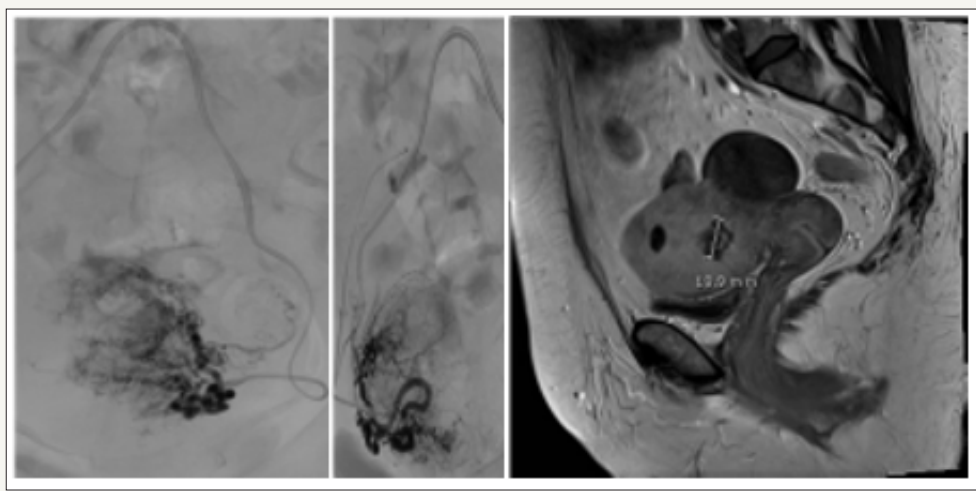

Figure 3: Bilateral uterine artery embolization (UAE). Significant shrinkage of fibroids after UAE at 6 months follow-up.

\section{Pelvic congestion syndrome (PCS)}

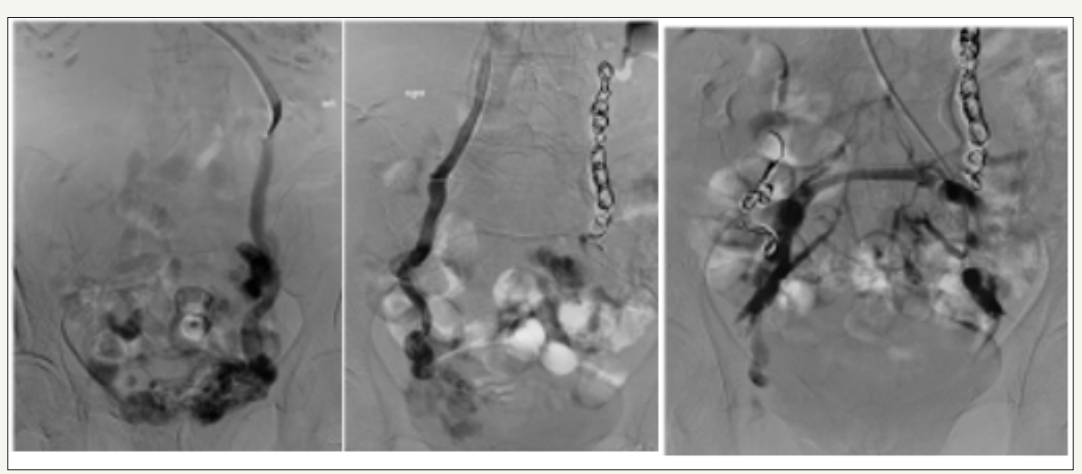

Figure 4: A woman with PCS during phlebography shows large incompetent bilateral ovarian veins, particularly on the left side crossing the midline and communicating with each other. Successful embolization of both ovarian veins with macro-coils.

PCS is a differential diagnosis for a patient with chronic pelvic pain (CPP), which is present in about $2-24 \%$ women from $18-50$ years of age [8]. The prevalence of PCS in patients with PCC is about 10-30\%, where no other obvious pathologies have been found [9]. Symptoms of PCS include CPP lasting at least 3-6 months, heaviness, pain during and after coitus, pain that can be exacerbated before or during menses, relived in supine position and can last several hours at a time. Diagnostic modalities are transvaginal color Doppler scanning, MR and contrast CT scanning. Although medical and surgical treatments are available for the treatment of PCS, pelvic venous embolization is currently considered the best treatment available for the treatment of PCS [10]. Ovarian and pelvic 
vein embolization has been recommended as the standard of care with grade $2 \mathrm{~B}$ recommendation by Society for vascular Surgery and American Venous Forum [11]. Refluxing ovarian and iliac veins can be approached from femoral, jugular, subclavian and brachial routes, all with high technical success and low complication rates [12]. Various embolic materials have been used in embolization for PCS including coils, foam (sclerotherapy), glue, plugs etc. However coil-embolization and/or foam sclerotherapy are most commonly used techniques. Clinical success rate has been described from about $70-100 \%$ in various studies although there lacks a well-designed large randomized control trial [10]. Complication rate is low and minor, a majority (around 15\%) of which is self-limiting post-embolization syndrome [13-15] (Figure 4).

\section{Conclusion}

The role of minimally invasive image guided interventions in gynecology is constantly evolving and is best defined in a multidisciplinary setting where decisions for interventions are jointly taken by the gynecologist and the interventional radiologist. Many of the procedures can be performed under local anesthesia or conscious sedation, in a day care basis in a minimally invasive manner with short hospital stay and very low complication rate. Most complications are minor and are either self-limiting or requires minimal medication/therapy. The role of image guided intervention in gynecology and women's health can be lifesaving, assisting in surgery by decreasing vascularity and blood loss in the operating field or significantly improve symptoms and quality of life.

\section{References}

1. Pelage JP, Le Dref O, Mateo, Soyer P, Jacob D, et al. (1998) Life-threatening primary postpartum hemorrhage: Treatment with selective arterial embolization. Radiology 208(2): 359-362.

2. Royal College of Obstetricians and Gynaecologists (2007) The role of emergency and elective interventional radiology in postpartum hemorrhage. Royal College of Obstetricians and Gynaecologists Good Practice Guideline No. 6. Royal College of Obstetricians and Gynaecologists, London.
3. Malik SN, Shams M (2012) Role of uterine artery embolization in the management of cervical cancer: Review article. J Cancer Sci Ther 4: 167169.

4. Baird D, Dunson DB, Hill MC, Cousins D, Schectman JM (2003) High cumulative invidence of uterine leiomyoma in black and white women: ultrasound evidence. Am J Obstet Gynecol 188(1): 100-107.

5. Donnez J, Jadoul P (2002) What are the implications of myomas on fertility? A need for a debate. Hum Reprod 17(6): 1424-1430.

6. https://www.rcog.org.uk/globalassets/documents / guidelines/23-12-2013_rcog_rcr_uae.pdf

7. Gupta JK, Sinha AS, Lumsden MA, Hickey M (2006) Uterine artery embolization for symptomatic uterine fibroids. Cochrane Database Syst Rev 16(5): CD005073.

8. Latthe P, Latthe M, Say L, Gülmezoglu M, Khan KS (2006) WHO systematic review of prevalence of chronic pelvic pain: a neglected reproductive health morbidity. BMC Public Health 6(6): 177.

9. Fassiadis N (2006) Treatment for pelvic congestion syndrome causing pelvic and vulvar varices. Int Angio 25(1): 1-3.

10. Borghi C, Dell'Atti L (2016) Pelvic congestion syndrome: the current state of the literature. Arch Gynecol Obstet 293(2): 291-301.

11. Gloviczki P, Comerota AJ, Dalsing MC, Eklof BG, Gillespie DL, et al. (2011) The care of patients with varicose veins and associated chronic venous diseases: clinical practice guidelines of the society for vascular surgery and the American venous forum. J Vasc Surg 53(5 Suppl): 2S-48S.

12. Freedman J, Ganeshan A, Crowe PM (2010) Pelvic congestion syndrome: the role of interventional radiology in the treatment of chronic pelvic pain. Postgrad Med J 86(1022): 704-710.

13. Hansrani V, Abbas A, Bhandari S, Caress AL, Seif M, et al. (2015) Transvenous occlusion of incompetent pelvic veins for chronic pelvic pain in women: a systematic review. Eur J Obstet Gynecol Reprod Biol 185: 156163.

14. Van der CJ, Kempen JA, Schultze LJ (2012) Embolization to treat pelvic congestion syndrome and vulval varicose veins. Int J Gynaecol Obstet 118(3): 227-230.

15. Laborda A, Medrano J, De Blas I, Urtiaga I, Carnevale FC, et al. (2013) Endovascular treatment of pelvic congestion syndrome: visual analog scale (vas) long-term follow-up clinical evaluation in 202 patients. Cardiovasc Intervent Radiol 36(4): 1006-10014.
Creative Commons Attribution 4.0 International License

For possible submissions Click Here

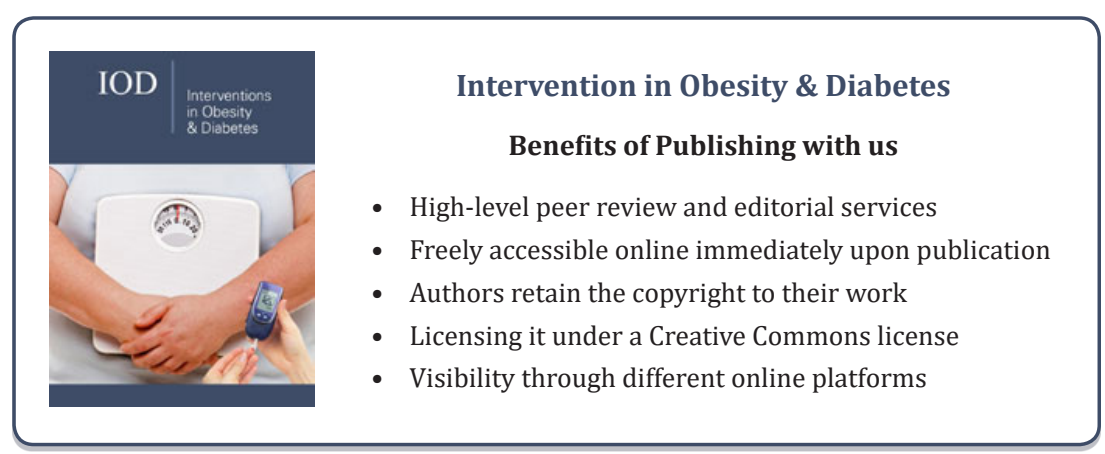

